OPEN ACCESS

Edited by: Jiaojian Wang,

University of Electronic Science and Technology of China, China

Reviewed by:

Qunlin Chen,

Southwest University, China

Fengmei Lu,

University of Macau, China

${ }^{*}$ Correspondence:

Yongcong Shao

budeshao@aliyun.com

Cuifeng Wang

15275228256@163.com

tThese authors have contributed equally to this work and share first

authorship

Specialty section:

This article was submitted to Brain Imaging and Stimulation,

a section of the journal

Frontiers in Human Neuroscience

Received: 14 May 2020 Accepted: 07 August 2020 Published: 12 October 2020

Citation:

Dai C, Zhang Y, Cai X, Peng Z, Zhang L, Shao $Y$ and Wang $C$ (2020)

Effects of Sleep Deprivation on

Working Memory: Change in Functional Connectivity Between the Dorsal Attention, Default Mode,

and Fronto-Parietal Networks.

Front. Hum. Neurosci. 14:360.

doi: 10.3389/fnhum.2020.00360

\section{Effects of Sleep Deprivation on Working Memory: Change in Functional Connectivity Between the Dorsal Attention, Default Mode, and Fronto-Parietal Networks}

\author{
Cimin Dai't, Ying Zhang ${ }^{21}$, Xiaoping Cai ${ }^{3}$, Ziyi Peng ${ }^{1}$, Liwei Zhang ${ }^{4}$, Yongcong Shao ${ }^{1,5 *}$ \\ and Cuifeng Wang ${ }^{6 *}$
}

${ }^{1}$ School of Psychology, Beijing Sport University, Beijing, China, ${ }^{2}$ The Eighth Medical Center of the General Hospital of People's Liberation Army, Beijing, China, ${ }^{3}$ Department of Cadraword 3 Division, General Hospital of People's Liberation Army, Beijing, China, ${ }^{4}$ Institute of Psychology, Chinese Academy of Sciences, Beijing, China, ${ }^{5}$ Suzhou Institute of Biomedical Engineering and Techology, Chinese Academy of Sciences, Suzhou, China, ${ }^{6}$ Department of Respiratory Medicine, Qingdao Huangdao People's Hospital, Qingdao, China

Sleep deprivation (SD) is very common in modern society and has a profound effect on cognitive function, in particular on working memory (WM). This type of memory is required for completion of many tasks and is adversely affected by SD. However, the cognitive neural mechanism by which SD affects WM, remains unclear. In this study, we investigated the changes in the brain network involved in WM after SD. Twentytwo healthy subjects underwent functional magnetic resonance imaging scan while in a state of resting wakefulness and again after $36 \mathrm{~h}$ of total SD and performed a WM task before each scanning session. Nineteen main nodes of the default mode network (DMN), dorsal attention network (DAN), fronto-parietal network (FPN), salience network (SN), and other networks were selected for functional analysis of brain network connections. Functional connectivity measures were computed between seed areas for region of interest (ROI)-to-ROI analysis and to identify patterns of ROI-to-ROI connectivity. The relationship between the significant changes in functional connectivity in the brain network and WM performance were then examined by Pearson's correlation analysis. WM performance declined significantly after SD. Compared with the awake state, the functional connectivity between DAN and DMN significantly increased after SD while that between FPN and DMN significantly decreased. Correlation analysis showed that the enhanced functional connectivity between DAN and DMN was negatively correlated with the decline in WM performance and that the decline in functional connectivity between FPN and DMN was positively correlated with decreased WM performance. These findings suggested that SD may affect WM by altering the functional connectivity among DMN, DAN, and FPN.

Keywords: sleep deprivation, working memory, functional connectivity, brain network, fMRI 


\section{INTRODUCTION}

Sleep is very important in both humans and animals. However, the pace and pressure of modern life is increasing, and an ever-increasing number of individuals are reporting sleep problems. Sleep deprivation (SD) has many negative physical and psychological effects, one of which is an adverse impact on cognitive function (Frenda et al., 2014; Boardman et al., 2018; Kusztor et al., 2019). Research on sleep disorders may help to improve our understanding of sleep and the negative outcomes associated with sleep disorders.

Working memory (WM) is a limited memory system that processes and temporarily stores information and plays an important role in many complex cognitive activities (Baddeley, 2010). It is also the basis for higher cognitive functions, including understanding, learning, and reasoning. The role of WM in advanced cognitive activities is important and much research effort has been focused on its composition and structure. Baddeley (2007) divided the WM system into three parts: the central executive system, which is the core component, and two other subordinate systems, the visuospatial sketchpad, which can maintain and operate visual and spatial information, and the phonological loop, which can passively store and actively retell voice information. Baddeley (2012) subsequently added another subordinate system, the episodic buffer, which can temporarily integrate multiple information from different coding systems. In addition to Baddeley's multi-component WM model, there are other WM models, including Cowan's embedded-processes model and Oberauer's concentric model (Cowan, 1999; Oberauer, 2002; Chein et al., 2003). However, the most widely accepted model is the multi-component model devised by Baddeley.

The prefrontal cortex (PFC) is an important brain region for central executive function and is the core subcomponent of WM (Jones and Harrison, 2001; Jemery and Volker, 2019). A large number of studies have shown that the anterior cingulate cortex (ACC) and PFC, which includes the ventrolateral PFC (VLPFC) and the dorsolateral PFC (DLPFC), contain areas that are important for WM (Jansma et al., 2000; Collette and Linden, 2002; Curtis and D'esposito, 2003; Bunge et al., 2005; Badre and Wagner, 2007). For example, the DLPFC plays an important role in task coordination and attention distribution in executive control (Blumenfeld and Ranganath, 2007). Furthermore, there are many studies on the relationship between WM and functional connectivity in the brain (Luerding et al., 2008; Roberts et al., 2017). Many functional magnetic resonance imaging (fMRI) studies on the mechanism of WM in the brain suggest that resting-state $\mathrm{fMRI}$ is an effective tool for exploring the neural mechanisms of WM (Heuvel and Pol, 2010; Biswal, 2012).

Sleep deprivation causes a decline in many cognitive functions, one of the most sensitive of which is WM (Chee et al., 2006; Preece, 2012; Martínez-Cancino et al., 2015). Behavioral studies of WM after SD found that the longer the duration of SD, the longer the response time and the lower the accuracy rate $(\mathrm{Lu}$, 2016; Tempesta et al., 2017; Harrington et al., 2018). fMRI studies have found that several brain regions, including the VLPFC, DLPFC, ACC, and posterior parietal cortex (PPC) are involved in processing of WM (Greicius et al., 2003; Owen et al., 2005;
Badre and Wagner, 2007; Wu et al., 2014) and that the DLPFC, ACC, and posterior parietal lobe (PPL) are associated with WM load (Cappell et al., 2010; Yoon et al., 2016). There are changes in the connections between these brain regions after $\mathrm{SD}$, as well as changes in the functional connectivity networks within these regions, which include the Default Mode Network, Dorsal Attention Network, Frontoparietal Network, and Salience Network (DMN, DAN, FPN, and SN, respectively) (Mu et al., 2005; Gujar et al., 2010; Havas et al., 2012). The changes in the functional connectivity in these brain regions may help us to understand the physiological basis of the effects of SD on WM. Therefore, these four brain function networks were our focus in this study.

Although there have many explorations of the neural mechanism involved in the decline in cognitive function and WM performance in sleep-deprived subjects, there is limited information on the changes in WM performance and the functional brain network after SD. The existing research has not clarified the impact of changes in network node functional connections. Analyses of WM based on a theoretical model should be closely related to the cognitive mechanism of attention but are rare. Therefore, this study selected the main nodes of the functional brain network that are closely related to WM to analyze the relationship between changes in the functional connections within these networks and the decrease in WM based on the WM model. Our research assumed that the mechanism of the decline in WM after SD was related to changes in functional connections between the FPN, DAN, and DMN.

\section{MATERIALS AND METHODS}

\section{Subjects}

The study participants were recruited by advertisements placed at Beihang University. The inclusion criteria were righthandedness, normal or corrected vision, normal cognitive function, and at least average intelligence (confirmed by Combined Raven Test score of $\geq 50 \%$ ). Participants were excluded if they had neurological or cardiovascular disease, cataract or glaucoma, pulmonary disease, hearing problems, as well as substance abusers. Participants also had to be free of sleep disorders. All participants were counseling to avoid smoking and drinking coffee and to aim for $8 \mathrm{~h}$ of regular sleep per day during the week before the experiment. Finally, 22 individuals were eligible to participate in the study. The study was approved by the ethics committee of The General Hospital of PLA (Beijing, China). All study participants fully understood the protocol and provided written informed consent before participating in the experiment.

\section{Experimental Paradigm}

The experiment was performed at Beihang University. All study participants had access to medical staff for rescue intervention at any time outside the laboratory for the duration of the study. For continuous behavioral monitoring, we matched participants with a partner to help them stay awake at night. Participants could 
not leave the laboratory after the start of SD and were escorted to the scanning room.

All the participants arrived at the laboratory at $4 \mathrm{pm}$ on day 1 and completed a demographic questionnaire and the Self-Rating Anxiety Scale, Self-Rating Depression Scale, and Pittsburgh Sleep Quality Index tests. At approximately $8 \mathrm{pm}$ on day 1, fMRI was performed when the subject was in a state of resting wakefulness (RW), after which WM tests were performed. After a routine nocturnal sleep period, total sleep deprivation (TSD) was started at 8 am on day 2 and ended at $8 \mathrm{pm}$ on day 3. The study participants were required to remain awake for the entire 36-h TSD session (Terán-Pérez et al., 2012). The participants underwent fMRI scanning at $8 \mathrm{pm}$ on day 3 and then repeated the WM tests. The experimental design is shown in Figure 1.

\section{WM Task Paradigm}

In recent decades, the $\mathrm{n}$-back paradigm has been widely used in the study of WM because the task is of moderate difficulty and sensitivity. This paradigm is often used to measure executive control in WM and to explore the neural mechanism of WM based on resting-state fMRI data (Martínez-Cancino et al., 2015; Soveri et al., 2017; Costers et al., 2020). The task requires the subject to monitor a stimulus sequence to determine if the stimulus currently presented is the same as that presented before several trials. So, participants need to timely monitor, update, and manipulate the information that is remembered (Seidman et al., 2006). Our study evaluated the WM performance of each subject through the 2-back task of visual WM. We adopted "Something Special" as the 2-back task with English letters as the external stimuli, although all the participants were native Chinese speakers. The participants received a series of visual letter stimuli on a computer screen and were asked to press the confirmation button on the keyboard as soon as possible, if the current letter was identical to that presented two trials back. The stimulus material included 15 upper case letters and 15 lower case letters (excluding letters similar to glyphs, such as L and M), a total of 122 letters with a letter size of $2.0 \times 2.0 \mathrm{~cm}$ (width $1.5^{\circ}$, height $1.5^{\circ}$ ), and a test time of about $5 \mathrm{~min}$. In the experiment, a prompt message (a white "+") was displayed in the center of
TABLE 1 | Demographic data, psychological traits, and sleep evaluation.

\begin{tabular}{lcccc}
\hline & RW state & TSD state & $\boldsymbol{t}$ & $\boldsymbol{p}$ \\
\hline Age (years) & $24.00 \pm 3.28$ & - & -- & -- \\
Male (n[\%]) & $22(100 \%)$ & - & -- & -- \\
BMl (kg/m²) & $22.82 \pm 1.98$ & - & -- & -- \\
Education (years) & $16.14 \pm 1.49$ & - & -- & -- \\
SAS & $20.36 \pm 10.81$ & - & -- & -- \\
SDS & $22.82 \pm 13.42$ & - & -- & -- \\
PSQI & $3.36 \pm 1.53$ & & & \\
Heart rate & $71.09 \pm 7.35$ & $71.73 \pm 7.86$ & -0.258 & 0.799 \\
Respiratory rate & $18.82 \pm 2.28$ & $18.18 \pm 2.02$ & 0.960 & 0.348 \\
Correct response per & $2.80 \pm 0.36$ & $2.47 \pm 0.31$ & 9.508 & $<0.0001$ \\
second $(W M)^{a}$ & & & &
\end{tabular}

t, paired t-test; BMI, Body mass index; SAS, Self-rating anxiety scale; SDS, Selfrating depression scale; PSQI, Pittsburgh sleep quality index; POMS, Profile of

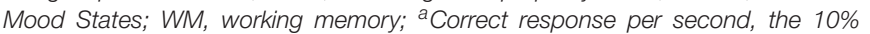
fastest correct trials of working memory.

the display for a duration of $200 \mathrm{~ms}$. After the prompt message had disappeared for $1 \mathrm{~s}$, letters were displayed in the center of the screen one by one with a duration of $400 \mathrm{~ms}$ for each letter. The stimulus interval was $1600 \mathrm{~ms}$. There were three 2back blocks, each consisting of 30 stimuli, including 10 target letters. Before each task block started, an introduction instructed the subject how to perform the task. Finally, the study used the correct response per second to assess WM performance. The WM task was performed out of the scan and immediately after the fMRI scan was completed. To calculate the correct response per second, the most rapid 10\% responses of each subject were selected as the most accurate test of WM, drawing lessons from the calculation method of the psychomotor vigilance test (PVT) indicator (Basner and Dinges, 2011; Chen et al., 2018).

\section{Resting State Paradigm}

All participants underwent structural MRI and fMRI twice, in the resting state on both occasions. During the scans, participants were requested to stay awake with eyes open, remain still, and not think about anything in particular.

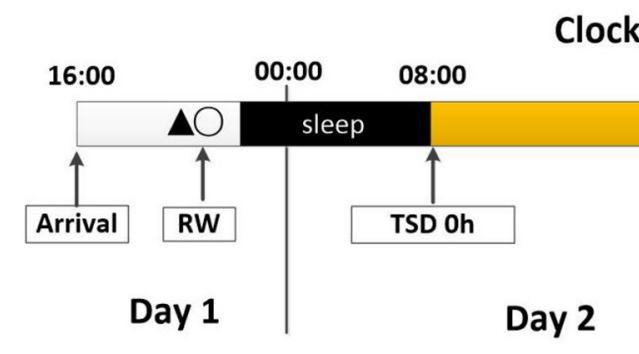

Clock time

$\Delta$ fMRI scanning

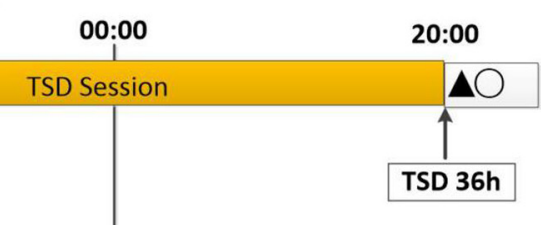

\section{Day 3}

\section{Behavioral test}

FIGURE 1 | Experimental design and protocol. All the participants enter the laboratory at 16:00 on Day 1, and then complete the regular test. At approximately 20:00 on Day 1, fMRI scanning under RW condition will be done, followed by behavioral tests (working memory). After a routine nocturnal sleep period, TSD started at 8:00 on Day 2 and ended at 20:00 at on Day 3. During the whole TSD session, the participants needed to keep awaking for 36 h. The participants performed fMRI scanning after TSD at 20:00 on Day 3, and then had behavioral tests (working memory). 


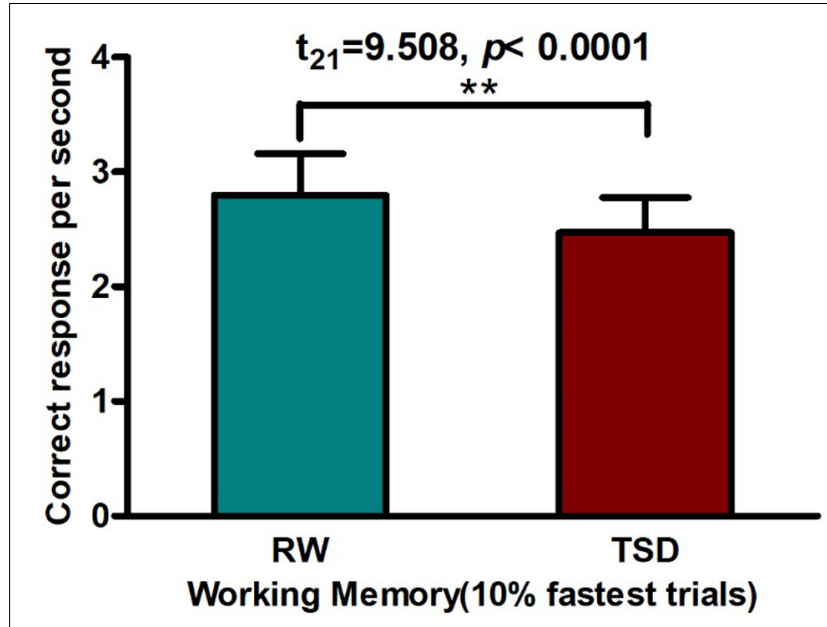

FIGURE 2 | Worse working memory performance contrasting in RW vs. TSD: After $36 \mathrm{~h}$ TSD, correct response per second decreased by a paired $t$-test $\left[{ }^{\star *} p<0.0001\right]$. TSD, total sleep deprivation; RW, rested wakefulness.

\section{Data Acquisition}

Standard static functional images and $\mathrm{T}^{*}$ weighted echo-planar image (EPI) sequences were obtained at 309th Hospital of PLA using a 3.0T Signa scanner (Siemens AG, Munich, Germany) with a birdcage RF head coil. The scanning protocol consists of (i) localization, (ii) T1-flair anatomy $(\mathrm{TR}=2 \mathrm{~s}$, $\mathrm{TE}=30 \mathrm{~ms}$, flip angle $=12^{\circ}, \mathrm{FoV}=256 \times 256 \mathrm{~mm}$, Sag slice number $=192$, voxel size $=1 \times 1 \times 1 \mathrm{~mm}$, no slice gap), and (iii) one resting-state session $\left(\mathrm{TR}=2 \mathrm{~s}, \mathrm{TE}=30 \mathrm{~ms}\right.$, flip angle $=90^{\circ}$, FoV $=256 \times 256 \mathrm{~mm}$, matrix $=64 \times 64$, Slice $=35$, Slice thickness $=3 \mathrm{~mm}$, Slice gap $=1 \mathrm{~mm})$. The scan duration was $480 \mathrm{~s}$. The subject was placed in the scanner with the head comfortably restricted by foam padding to reduce head movement and earplugs to attenuate the noise of the scanner. During the resting-state scan, all participants was asked to keep their eyes open, lie as still as possible, and not think about anything too specific. A pulse oximeter was attached to a finger to record heart activity. All study participants also wore pressure
TABLE 2 | DMN, DAN, FPN and SN networks main node and location in resting Networks.

\begin{tabular}{|c|c|c|c|}
\hline network & ROI & ROI name & MNI center \\
\hline \multirow[t]{4}{*}{ DMN } & ILP & Left paracentral lobule & $-39,-77,33$ \\
\hline & MPFC & Medial prefrontal cortex & $1,55,-3$ \\
\hline & PCC & Posterior cingulate & $1,-61,38$ \\
\hline & rLP & Right paracentral lobule & $47,-67,29$ \\
\hline \multirow[t]{4}{*}{ DAN } & IFEF & Left frontal eye field & $-27,-9,64$ \\
\hline & IIPS & Left intraparietal sulcus & $-39,-43,52$ \\
\hline & rFEF & Right frontal eye field & $30,-6,64$ \\
\hline & rIPS & Right intraparietal sulcus & $39,-42,54$ \\
\hline \multirow[t]{4}{*}{ FPN } & ILPFC & Left lateral prefrontal cortex & $-43,33,28$ \\
\hline & IPPC & Left posterior parietal cortex & $-46,-58,49$ \\
\hline & rLPFC & Right lateral prefrontal cortex & $41,38,30$ \\
\hline & rPPC & Right posterior parietal cortex & $52,-52,45$ \\
\hline \multirow[t]{7}{*}{ SN } & $\mathrm{ACC}$ & Anterior cingulate & $0,22,35$ \\
\hline & IAlnsula & Left anterior insula & $-44,13,1$ \\
\hline & IRPFC & Left rostrolateral prefrontal cortex & $-32,45,27$ \\
\hline & ISMG & Left supramarginal gyrus & $-60,-39,31$ \\
\hline & rAlnsula & Right anterior insula & $47,14,0$ \\
\hline & rRPFC & Right rostrolateral prefrontal cortex & $32,46,27$ \\
\hline & rSMG & Right supramarginal gyrus & $62,-35,32$ \\
\hline
\end{tabular}

$D A N$, dorsal attention network; DMN, default mode network; FPN, fronto-parietal network; SN, salience network.

bands around the waist to record breathing activity. The cardiac and respiratory signals collected were synchronized with the fMRI data to ensure that physiological changes would be removed during regression analysis. To ensure that participants did not fall asleep during the scan, the researcher monitored them using a camera and reminded them of the need to stay awake through a microphone before each scan if necessary. After each trial, the participants were asked if they had remained awake and all reported that they had done so.

\section{Data Preprocessing}

The functional images were preprocessed using SPM 12 (University College London ${ }^{1}$ ) and CONN toolbox v17a (Neuroimaging Informatics Tools and Resources

\footnotetext{
${ }^{1}$ http://www.fil.ion.ucl.ac.uk/spm/
}
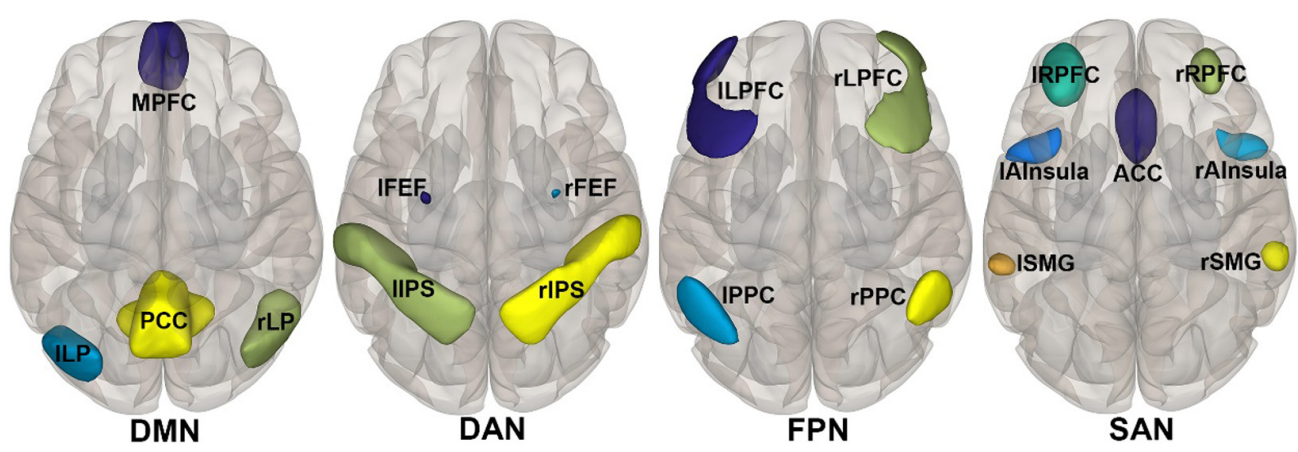

FIGURE 3 | DMN, DAN, FPN, and SAN node location in resting Networks: According to the network template in CONN, 19 main nodes of the four networks related to working memory are selected, and their locations are shown in the figure. Their specific names and coordinates are shown in Table 2. DAN, dorsal attention network; DMN, default mode network; FPN, fronto-parietal network; SAN, salience network. 
Clearinghouse $^{2}$ ) software implemented in the 2016 version of MATLAB. CONN is a MATLAB-based cross-platform software for computing and analyzing functional connections in brain regions based on fMRI signals. This software has been widely used and its reliability has been well documented (Whitfield-Gabrieli and Nieto-Castanon, 2012). The first 10 volumes of the functional time series of each epoch were discarded so that the signal was stable, and participants were accustomed to scanning noise. Rotation and movement of all participants was within $2 \mathrm{~mm}$ or 2 degrees in the $\mathrm{x}$, $y$, and $z$ planes, respectively. Next, slice-timing and headmotion corrections were performed. Volumes were normalized to the standard EPI template in the Montreal Institute of Neuroscience space and restored to $3 \times 3 \times 3 \mathrm{~mm}$. The resulting images were spatially smoothed with a $6 \mathrm{~mm}$ full width at half-maximum Gaussian kernel (Lu et al., 2020). In order to minimize the impact of motion and physiological noise factors, such as cardiac and respiratory signals, the CompCor approach was used for spatial and temporal preprocessing to define and remove confounds in the BOLD signal (Behzadi et al., 2007).

\section{Functional Connectivity Analysis}

We scanned the ROI-ROI connectivity matrix, tested the hypotheses, and visualized data using the CONN toolbox implemented in the 2016 version of MATLAB version and SPM12. Nineteen ROIs from the DAN, DMN, FPN, and $\mathrm{SN}$ in the brain were drawn from the CONN software

${ }^{2}$ https://www.nitrc.org/projects/conn template (conn/rois/networks.nii). All the ROIs in the four networks were imported into the CONN toolbox. Functional connectivity measures were computed between seed areas for ROI-to-ROI analysis and to identify patterns of ROI-toROI connectivity. Partial correlation was used to estimate the functional connectivity between two nodes (Li et al., 2020). We compared functional connectivity between the RW and TSD scans using two-tailed paired $t$-tests. The significance of ROIto-ROI connectivity was determined based on FDR-corrected $p$-Values. The analysis of TSD versus RW connectivity was set at an FDR-corrected $p$-Value of 0.05 for the voxel-level height threshold in both ROI-to-ROI and seed ROI tests.

\section{Behavioral Correlation}

The relationship between the decline in WM and changes in functional connectivity were examined using correlation analysis. Correlations between changes in the correct response time (the $10 \%$ most rapid WM trials) and altered functional connectivity before and after SD were calculated. The significance level was kept at $p<0.05$ to obtain the correlation between ROI and behavior.

\section{RESULTS}

\section{Physiological Data}

Table 1 shows the demographic data, psychological features, and sleep characteristics of the study participants. All participants were monitored for breathing and heart rate during the fMRI scans. The paired $t$-test was used to compare the mean respiration

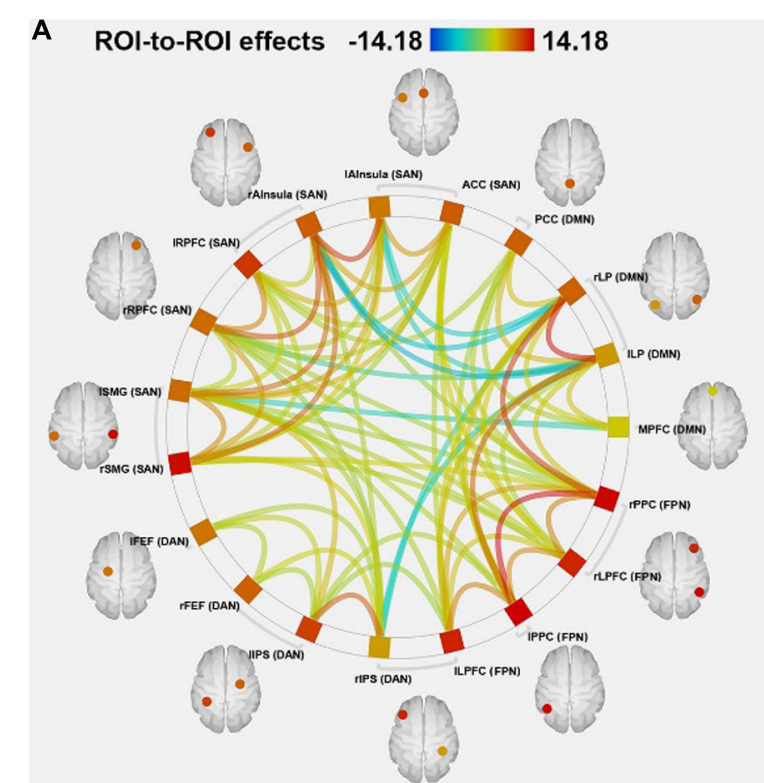

RW
B
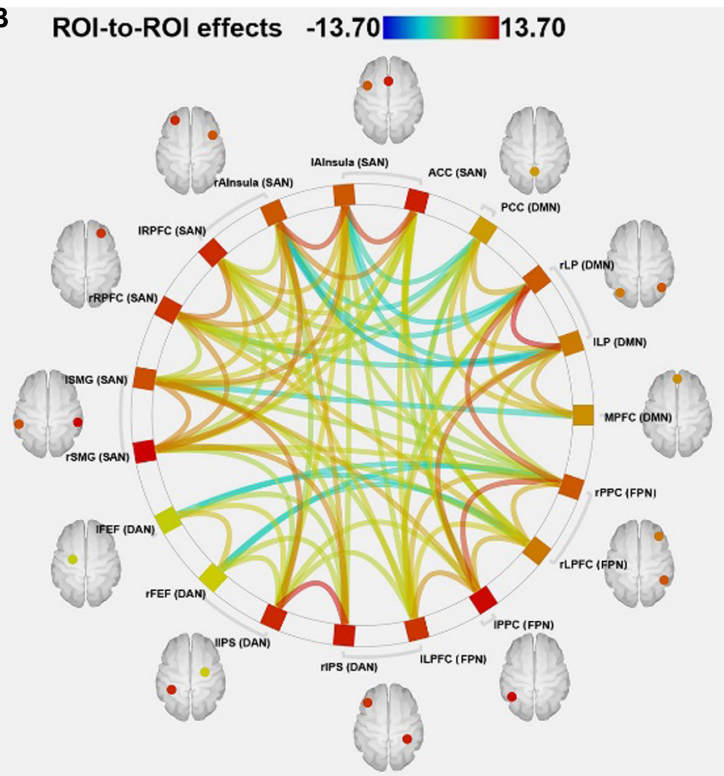

TSD

FIGURE 4 | ROI-to-ROI connectivity of four networks in panels (A) RW and (B) TSD scans [p < 0.05 FDR set wise corrected for all comparisons across the entire network]. ROI, region of interest; TSD, total sleep deprivation; RW, rested wakefulness. DAN, dorsal attention network; DMN, default mode network; FPN, fronto-parietal network; SAN, salience network. 
TABLE 3 | Resting state functional connectivity ROI pairs within- and betweenDAN, DMN, FPN, and SN network for RW scans (t-test).

\begin{tabular}{|c|c|c|c|}
\hline Network & ROI-to-ROI & $t$ & p-FDR \\
\hline \multirow[t]{21}{*}{ Within-SN } & ACC-IAInsula & 7.3 & 0.0000 \\
\hline & ACC-IRPFC & 3.89 & 0.0022 \\
\hline & ACC-ISMG & 5.76 & 0.0001 \\
\hline & ACC-rAlnsula & 6.54 & 0.0000 \\
\hline & ACC-rRPFC & 3.18 & 0.0089 \\
\hline & ACC-rSMG & 2.92 & 0.0148 \\
\hline & IAlnsula-IRPFC & 6.02 & 0.0000 \\
\hline & IAlnsula-ISMG & 6.58 & 0.0000 \\
\hline & IAlnsula-rAlnsula & 11.91 & 0.0000 \\
\hline & IAlnsula-rRPFC & 3.51 & 0.0053 \\
\hline & IAlnsula-rSMG & 5.09 & 0.0002 \\
\hline & IRPFC-ISMG & 4.45 & 0.0013 \\
\hline & IRPFC-rAlnsula & 4.06 & 0.0025 \\
\hline & IRPFC-rRPFC & 8.25 & 0.0000 \\
\hline & IRPFC-rSMG & 3.85 & 0.0028 \\
\hline & ISMG-rAlnsula & 9.51 & 0.0000 \\
\hline & ISMG-rRPFC & 3.77 & 0.0028 \\
\hline & ISMG-rSMG & 7.87 & 0.0000 \\
\hline & rAlnsula-rRPFC & 10.58 & 0.0000 \\
\hline & rAlnsula-rSMG & 9.67 & 0.0000 \\
\hline & rRPFC-rSMG & 6.96 & 0.0000 \\
\hline \multirow[t]{6}{*}{ Within-FPN } & ILPFC-IPPC & 8.47 & 0.0000 \\
\hline & ILPFC-rLPFC & 5.30 & 0.0001 \\
\hline & ILPFC-rPPC & 6.79 & 0.0000 \\
\hline & IPPC-rLPFC & 7.88 & 0.0000 \\
\hline & IPPC-rPPC & 14.18 & 0.0000 \\
\hline & rLPFC-rPPC & 8.58 & 0.0000 \\
\hline \multirow[t]{6}{*}{ Within-DMN } & ILP-MPFC & 4.71 & 0.0003 \\
\hline & ILP-PCC & 5.48 & 0.0001 \\
\hline & ILP-rLP & 13.49 & 0.0000 \\
\hline & MPFC-PCC & 3.30 & 0.0154 \\
\hline & MPFC-rLP & 5.36 & 0.0005 \\
\hline & PCC-rLP & 5.63 & 0.0002 \\
\hline \multirow[t]{6}{*}{ Within-DAN } & IFEF-IIPS & 4.37 & 0.0048 \\
\hline & IFEF-rFEF & 3.56 & 0.0168 \\
\hline & IFEF-rIPS & 3.04 & 0.0371 \\
\hline & IIPS-rFEF & 3.12 & 0.0187 \\
\hline & IIPS-rIPS & 10.22 & 0.0000 \\
\hline & rFEF-rIPS & 4.57 & 0.0030 \\
\hline \multirow[t]{13}{*}{ SN-FPN } & rPPC-rAlnsula & 2.36 & 0.0458 \\
\hline & rSMG-rLPFC & 3.58 & 0.0040 \\
\hline & ACC-IPPC & 4.54 & 0.0005 \\
\hline & ISMG-IPPC & 3.73 & 0.0028 \\
\hline & ISMG-rLPFC & 3.50 & 0.0043 \\
\hline & rSMG-rPPC & 4.71 & 0.0003 \\
\hline & ISMG-ILPFC & 3.31 & 0.0055 \\
\hline & ISMG-rPPC & 2.89 & 0.0122 \\
\hline & rRPFC-rLPFC & 3.54 & 0.0062 \\
\hline & rRPFC-rPPC & 2.98 & 0.0161 \\
\hline & ACC-rPPC & 4.74 & 0.0004 \\
\hline & ACC-rLPFC & 4.72 & 0.0004 \\
\hline & ACC-ILPFC & 3.49 & 0.0050 \\
\hline
\end{tabular}

TABLE 3 | Continued

\begin{tabular}{|c|c|c|c|}
\hline Network & ROI-to-ROI & $t$ & p-FDR \\
\hline \multirow[t]{7}{*}{ SN-DMN } & rAlnsula-rLP & -5.74 & 0.0000 \\
\hline & rAlnsula-ILP & -5.13 & 0.0001 \\
\hline & ISMG-MPFC & -3.33 & 0.0055 \\
\hline & |Alnsula-ILP & -3.81 & 0.0030 \\
\hline & IAlnsula-rLP & -2.96 & 0.0169 \\
\hline & rRPFC-rLP & -2.38 & 0.0479 \\
\hline & IRPFC-PCC & 3.51 & 0.0053 \\
\hline \multirow[t]{5}{*}{ SN-DAN } & rAlnsula-rIPS & 3.23 & 0.0080 \\
\hline & rSMG-rIPS & 4.77 & 0.0004 \\
\hline & ISMG-IIPS & 5.87 & 0.0000 \\
\hline & ISMG-rIPS & 3.12 & 0.0078 \\
\hline & rRPFC-rIPS & 2.43 & 0.0479 \\
\hline \multirow[t]{10}{*}{ FPN-DMN } & IPPC-rLP & 9.86 & 0.0000 \\
\hline & ILPFC-rLP & 5.62 & 0.0001 \\
\hline & rPPC-rLP & 5.22 & 0.0001 \\
\hline & rLPFC-rLP & 3.02 & 0.0129 \\
\hline & PCC-rPPC & 3.59 & 0.0038 \\
\hline & rPPC-ILP & 11.68 & 0.0000 \\
\hline & IPPC-ILP & 5.31 & 0.0001 \\
\hline & ILPFC-ILP & 4.80 & 0.0003 \\
\hline & PCC-IPPC & 3.06 & 0.0119 \\
\hline & ILP-rLPFC & 4.65 & 0.0005 \\
\hline \multirow[t]{2}{*}{ FPN-DAN } & IPPC-IIPS & 3.31 & 0.0075 \\
\hline & ILPFC-\|PS & 2.65 & 0.0334 \\
\hline \multirow[t]{2}{*}{ DAN-DMN } & rIPS-rLP & -3.98 & 0.0031 \\
\hline & rIPS-ILP & -2.75 & 0.0269 \\
\hline
\end{tabular}

FDR, false discovery rate; $R O I$, region of interest; $R W$, rested wakefulness. $p<0.05$ FDR set wise corrected for all comparisons across the entire network. DAN, dorsal attention network; DMN, default mode network; FPN, fronto-parietal network; SN, salience network.

and heart rate values in each subject before and after SD. No difference in the heart rate $(t=-0.258, p>0.05)$ or respiration rate $(t=0.960, p>0.05)$ was found between the RW and TSD conditions (Table 1).

\section{Changes in WM Performance Before and After SD}

Using a paired $t$-test, we found that SD led to a significant decline in WM $\left(t_{21}=9.508, p<0.0001\right.$, see Figure 2 and Table 1), which is consistent with previous studies (Drummond et al., 2012; Martínez-Cancino et al., 2015; Lu, 2016; Tempesta et al., 2017; Harrington et al., 2018).

\section{Resting Networks}

According to the CONN template, 19 brain regions in the DMN, DAN, FPN, and SN were selected as ROIs. The DMN includes the left and right paracentral lobules (ILP and rLP), medial prefrontal cortex (MPFC), and posterior cingulate cortex (PCC); the DAN includes the left and right frontal eye fields, left intraparietal sulcus, and right intraparietal sulcus (rIPS); the FPN includes the left lateral prefrontal cortex, right lateral prefrontal cortex (rLPFC), left and right posterior parietal cortices; and the SN includes the ACC, left and right anterior insulae, left and right 
TABLE 4 | Resting state functional connectivity ROI pairs within- and betweenDAN, DMN, FPN, and SN network for TSD scans (t-test).

\begin{tabular}{|c|c|c|c|}
\hline Network & ROI-to-ROI & $t$ & p-FDR \\
\hline \multirow[t]{21}{*}{ Within-SN } & ACC-IAlnsula & 11.44 & 0.0000 \\
\hline & ACC-IRPFC & 4.03 & 0.0018 \\
\hline & ACC-ISMG & 3.73 & 0.0028 \\
\hline & ACC-rAlnsula & 7.87 & 0.0000 \\
\hline & ACC-rRPFC & 3.83 & 0.0025 \\
\hline & ACC-rSMG & 4.12 & 0.0017 \\
\hline & IAlnsula-IRPFC & 6.02 & 0.0000 \\
\hline & IAlnsula-ISMG & 7.79 & 0.0000 \\
\hline & IAlnsula-rAlnsula & 11.61 & 0.0000 \\
\hline & IAlnsula-rRPFC & 4.70 & 0.0003 \\
\hline & IAlnsula-rSMG & 5.18 & 0.0001 \\
\hline & IRPFC-ISMG & 5.48 & 0.0001 \\
\hline & IRPFC-rAlnsula & 3.88 & 0.0026 \\
\hline & IRPFC-rRPFC & 8.92 & 0.0000 \\
\hline & IRPFC-rSMG & 3.39 & 0.0072 \\
\hline & ISMG-rAlnsula & 4.30 & 0.0010 \\
\hline & ISMG-rRPFC & 3.42 & 0.0046 \\
\hline & ISMG-rSMG & 8.12 & 0.0000 \\
\hline & rAlnsula-rRPFC & 7.72 & 0.0000 \\
\hline & rAlnsula-rSMG & 8.66 & 0.0000 \\
\hline & rRPFC-rSMG & 8.07 & 0.0000 \\
\hline \multirow[t]{6}{*}{ Within-FPN } & ILPFC-IPPC & 7.51 & 0.0000 \\
\hline & ILPFC-rLPFC & 4.90 & 0.0003 \\
\hline & ILPFC-rPPC & 6.21 & 0.0000 \\
\hline & IPPC-rLPFC & 6.50 & 0.0000 \\
\hline & IPPC-rPPC & 11.36 & 0.0000 \\
\hline & rLPFC-rPPC & 6.30 & 0.0000 \\
\hline \multirow[t]{6}{*}{ Within-DMN } & ILP-MPFC & 6.18 & 0.0000 \\
\hline & ILP-PCC & 6.52 & 0.0000 \\
\hline & ILP-rLP & 13.70 & 0.0000 \\
\hline & MPFC-PCC & 5.60 & 0.0001 \\
\hline & MPFC-rLP & 6.86 & 0.0000 \\
\hline & PCC-rLP & 5.35 & 0.0002 \\
\hline \multirow[t]{6}{*}{ Within-DAN } & |FEF-||PS & 4.31 & 0.0028 \\
\hline & IFEF-rFEF & 5.42 & 0.0004 \\
\hline & IFEF-rIPS & 3.03 & 0.0230 \\
\hline & IIPS-rFEF & 4.11 & 0.0018 \\
\hline & IIPS-rIPS & 13.56 & 0.0000 \\
\hline & rFEF-rIPS & 3.69 & 0.0062 \\
\hline \multirow[t]{13}{*}{ SN-FPN } & ACC-ILPFC & 4.64 & 0.0006 \\
\hline & ACC-IPPC & 2.60 & 0.0302 \\
\hline & ACC-rLPFC & 4.88 & 0.0005 \\
\hline & |Alnsula-|LPFC & 3.80 & 0.0021 \\
\hline & rSMG-rLPFC & 4.11 & 0.0012 \\
\hline & IRPFC-ILPFC & 5.04 & 0.0002 \\
\hline & ISMG-IPPC & 6.43 & 0.0000 \\
\hline & ISMG-ILPFC & 3.27 & 0.0060 \\
\hline & ISMG-rPPC & 2.96 & 0.0113 \\
\hline & rAlnsula-rLPFC & 3.41 & 0.0053 \\
\hline & ACC-rPPC & 3.52 & 0.0041 \\
\hline & rRPFC-rLPFC & 5.79 & 0.0000 \\
\hline & rRPFC-rPPC & 2.41 & 0.0450 \\
\hline
\end{tabular}

(Continued)
TABLE 4 | Continued

\begin{tabular}{|c|c|c|c|}
\hline Network & ROI-to-ROI & $t$ & p-FDR \\
\hline & rSMG-rPPC & 4.07 & 0.0012 \\
\hline \multirow[t]{10}{*}{ SN-DMN } & |Alnsula-ILP & -3.94 & 0.0017 \\
\hline & IAlnsula-PCC & -2.50 & 0.0311 \\
\hline & IAlnsula-rLP & -2.64 & 0.0248 \\
\hline & IRPFC-PCC & 3.03 & 0.0142 \\
\hline & ISMG-MPFC & -3.53 & 0.0040 \\
\hline & rAlnsula-ILP & -3.32 & 0.0058 \\
\hline & rAlnsula-PCC & -2.58 & 0.0283 \\
\hline & rAlnsula-rLP & -5.32 & 0.0001 \\
\hline & rRPFC-PCC & 2.71 & 0.0271 \\
\hline & rRPFC-rLP & -2.70 & 0.0271 \\
\hline \multirow[t]{8}{*}{ SN-DAN } & |Alnsula-||PS & 4.73 & 0.0003 \\
\hline & |Alnsula-r|PS & 3.45 & 0.0043 \\
\hline & ISMG-IIPS & 8.92 & 0.0000 \\
\hline & ISMG-rIPS & 3.95 & 0.0019 \\
\hline & rAlnsula-IIPS & 2.29 & 0.0491 \\
\hline & rAlnsula-rIPS & 6.61 & 0.0000 \\
\hline & rSMG-rIPS & 7.79 & 0.0000 \\
\hline & rSMG-IIPS & 2.97 & 0.0132 \\
\hline \multirow[t]{8}{*}{ FPN-DMN } & ILPFC-ILP & 3.15 & 0.0086 \\
\hline & rPPC- ILP & 10.79 & 0.0000 \\
\hline & IPPC-ILP & 4.63 & 0.0004 \\
\hline & IPPC-PCC & 2.47 & 0.0445 \\
\hline & rLPFC-PCC & 2.83 & 0.0181 \\
\hline & ILPFC- rLP & 5.50 & 0.0001 \\
\hline & IPPC-rLP & 9.55 & 0.0000 \\
\hline & rPPC-rLP & 3.77 & 0.0034 \\
\hline \multirow[t]{6}{*}{ FPN-DAN } & IPPC-IIPS & 3.94 & 0.0019 \\
\hline & ILPFC-\|PS & 3.69 & 0.0035 \\
\hline & rLPFC-IFEF & -3.80 & 0.0027 \\
\hline & rLPFC-rFEF & -3.03 & 0.0126 \\
\hline & rPPC-IFEF & -3.63 & 0.0035 \\
\hline & rPPC-rFEF & -3.68 & 0.0035 \\
\hline
\end{tabular}

FDR, false discovery rate; $R O I$, region of interest; TSD, total sleep deprivation. $p<0.05$ FDR set wise corrected for all comparisons across the entire network. DAN, dorsal attention network; DMN, default mode network; FPN, fronto-parietal network; SN, salience network.

rostrolateral prefrontal cortices, and left and right supramarginal gyri. Figure 3 shows the abbreviated names and locations of the 19 nodes. Their specific names and coordinates are shown in Table 2. The functional connections within and between the DAN, DMN, FPN and SAN in the RW and TSD states are shown in Figure 4 and Tables 3, 4.

\section{Changes in Four Brain Network Connections Before and After SD}

After TSD, the functional connectivity was higher between DAN and DMN (rIPS-rLP) $\left(t_{21}=4.22, p=0.0045\right.$, FDR-corrected) and lower between FPN and DMN (rLPFC-rLP) $\left(t_{21}=-4.11\right.$, $p=0.0045$, FDR-corrected) when compared with the RW condition. The results are shown in Figure 5 and Table 5. 


\section{Correlations Between Changes in Strength of Connectivity and WM}

In order to explore the relationship between changes in functional connectivity and WM, we calculated the correlation between the change in correct responses per second of WM and that of the two functional connectivity before and after SD. The change in the number of correct responses per second was negatively correlated with increased functional connectivity of rIPS-rLP (DAN-DMN) and positively correlated with decreased functional connectivity of rLPFC-rLP (FPN-DMN). The results are shown in Figure 6.

\section{DISCUSSION}

Our study analyzed functional connectivity in the resting state of healthy subjects before and after SD. We found that functional connectivity increased between the DAN and DMN after SD and decreased between the FPN and DMN networks. Moreover, there was a significant positive relationship between enhancement of functional connectivity between the DAN and DMN and the decline in WM performance and a significant negative relationship between decreased functional connectivity between the FPN and DMN and the decline in WM performance. This finding indicates that enhanced connectivity between the DAN and DMN may have an important effect on the decline in WM after SD.

The main finding of this study was that SD enhances the functional connectivity between DAN and DMN and that this change is significantly related to a decline in WM performance. From the perspective of the WM model, DAN should be the core brain network for processing and maintaining visuospatial sketchpad (Fairhall et al., 2009; Tamber-Rosenau et al., 2018). The main function of DAN is to provide top-down attention to the outside world and participate in the completion of exogenous

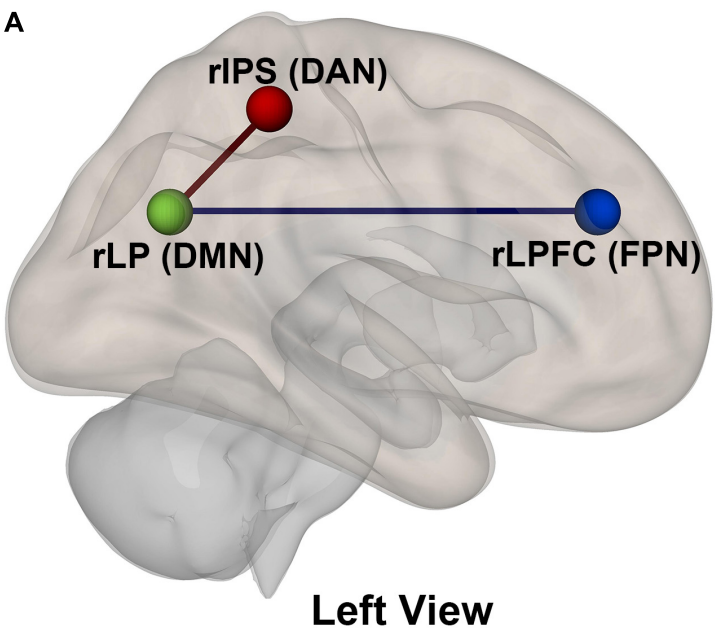

B
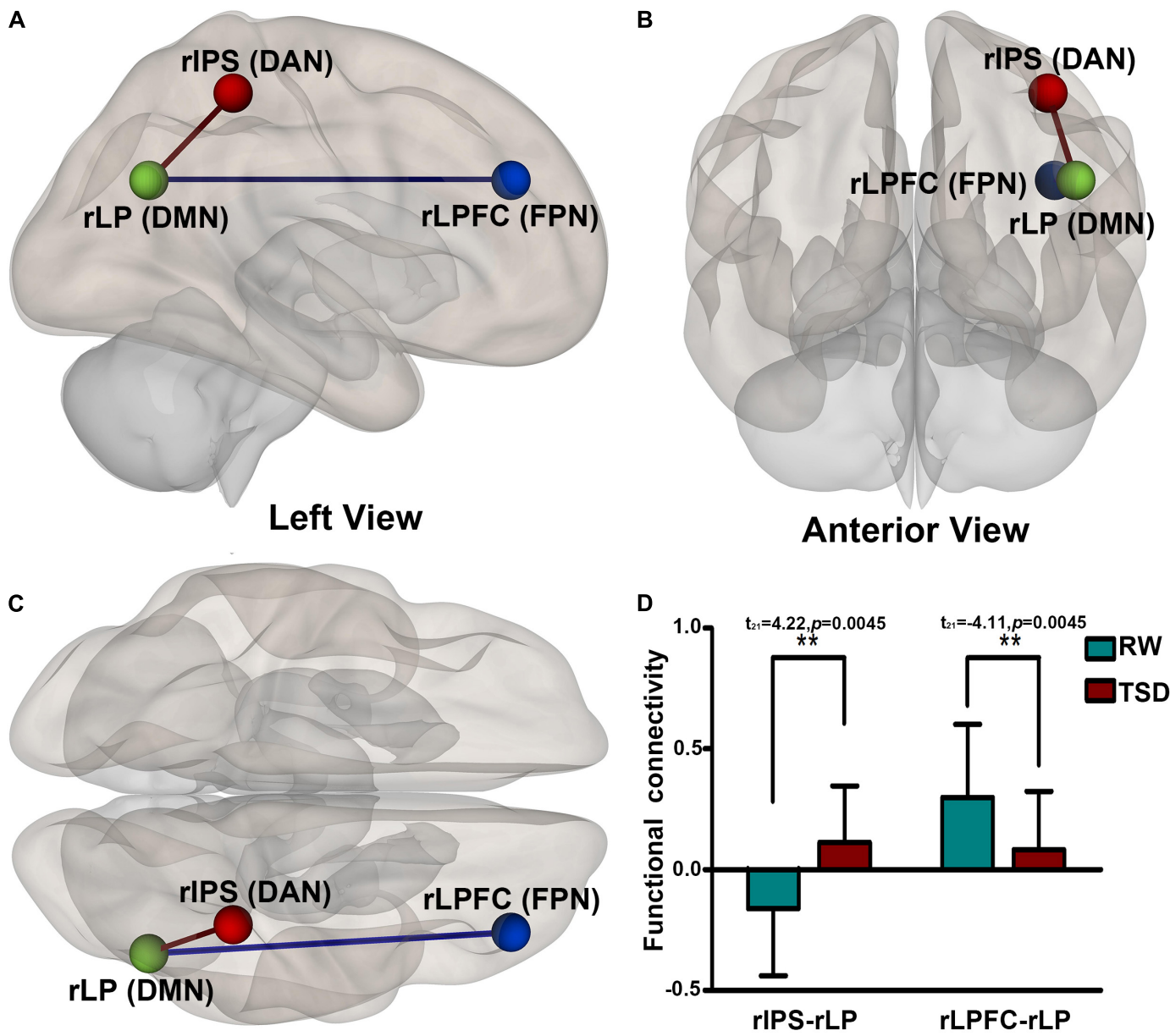

Superior view

FIGURE 5 | Alerted ROI-to-ROI functional connectivity of networks contrasting in RW vs. TSD scans: Comparing to RW scan, functional connectivity of rlPS-rLP (DAN-DMN) is higher (the red line), and functional connectivity of rLPFC-rLP (FPN-DMN) is lower (the blue line). All the results are shown at panels (A) left, (B) anterior, (C) Superior view, (D) shows the effect size of functional connectivity. [ ${ }^{\star *} p<0.05$ FDR set wise corrected for all comparisons across the entire network]. ROI, region of interest; TSD, total sleep deprivation; RW, rested wakefulness; rIPS, right intraparietal sulcus; DAN, dorsal attention network; rLP, right paracentral lobule; DMN, default mode network; rLPFC, right lateral prefrontal cortex; FPN, fronto-parietal network. 
TABLE 5 | ROI-to-ROI functional connectivity statistics of network: comparisons between RW and TSD scans ( $t$-test).

\begin{tabular}{lccc}
\hline Network & ROI-to-ROI & $\boldsymbol{t}$ & p-FDR \\
\hline DAN-DMN & rIPS-rLP & 4.22 & 0.0045 \\
FPN-DMN & rLPFC-rLP & -4.11 & 0.0045 \\
\hline
\end{tabular}

$R O I$, region of interest; FDR, false discovery rate; RW, rested wakefulness; TSD, total sleep deprivation. $p<0.005$ FDR set wise corrected for all comparisons across the entire network. DAN, dorsal attention network; DMN, default mode network; FPN, fronto-parietal network; SN, salience network.

tasks (Ptak and Schnider, 2010; Fornito et al., 2012). The main function of DMN is the individual's continuous attention to self, which antagonizes the function of DAN (Lei et al., 2013). DMN mainly includes the PFC, PCC, and ACC, which is the neural basis of the self and is related to maintaining the awake state (Moran et al., 2013). DMN is very special among brain networks because it is often more activated in the resting state than in the task state (Qin and Northoff, 2011). One possible explanation for this is that the DMN can interfere with attention to specific tasks (Schilbach et al., 2008). The enhanced functional connection between the DAN and DMN found in this study indicates that SD reduces the ability of the individual to distinguish between internal and external sources, which may result in inefficient allocation of attention resources. Moreover, there was a significant negative relationship between the enhanced connectivity between the DAN and DMN and the change in WM scores, confirming that changes in brain network connectivity do affect cognitive function in humans.

Another main finding of this study was that the functional connectivity between FPN and DMN declines after SD, which is in accordance with previous research showing that the functional connectivity of DMN and its anti-networks declines after SD
(Gujar et al., 2010; Havas et al., 2012). Further analysis found a significant positive relationship between this decline and changes in WM performance. FPN does not have left-right symmetry; hence, we need to distinguish the right FPN from the left. The left FPN is mainly related to verbal expression and memory (Zhu et al., 2017), and the right FPN basically participates in any task that requires cognitive control, such as WM and activity suppression (Seo et al., 2012). The FPN is considered a central actuator in the WM model and responsible for the macrocontrol of WM information processing (Sakagami and Tsutsui, 1999; Jones and Harrison, 2001; Wang et al., 2010; Jemery and Volker, 2019). There is a difference between DAN and FPN in terms of WM tasks that require constant monitoring and manipulation of information. In short, DAN is mainly involved in information manipulation, while FPN plays a more important role in information monitoring (Champod and Petrides, 2010).

Our results are in line with those of many other behavioral studies (Van et al., 2012; Lu, 2016; Tempesta et al., 2017; Harrington et al., 2018) but not all of them. Dunsmoor et al. (2015) found that sleep could selectively effect memory of related events but that sleep is not necessary to strengthen memory. The time interval between the experience itself and recall may be more important. Gerhardsson et al. (2019) found no impairment of WM in sleep-deprived elderly subjects but found differences in effects of SD on WM in people of different ages. In an electroencephalographic (EEG) study, memory performance worsened after SD and there was a significant change in the correlation between the left and right PFC, indicating that SD impairs memory by affecting the PFC (Li et al., 2014). This result is consistent with the finding by Yeung et al. (2018) in a study using near-infrared spectroscopy (NIRS) that the frontal cortex was inhibited after SD. In studies of event-related potentials, it was found that performance on memory tasks decreased after $\mathrm{SD}$ and that there was a change in the values of some EEG
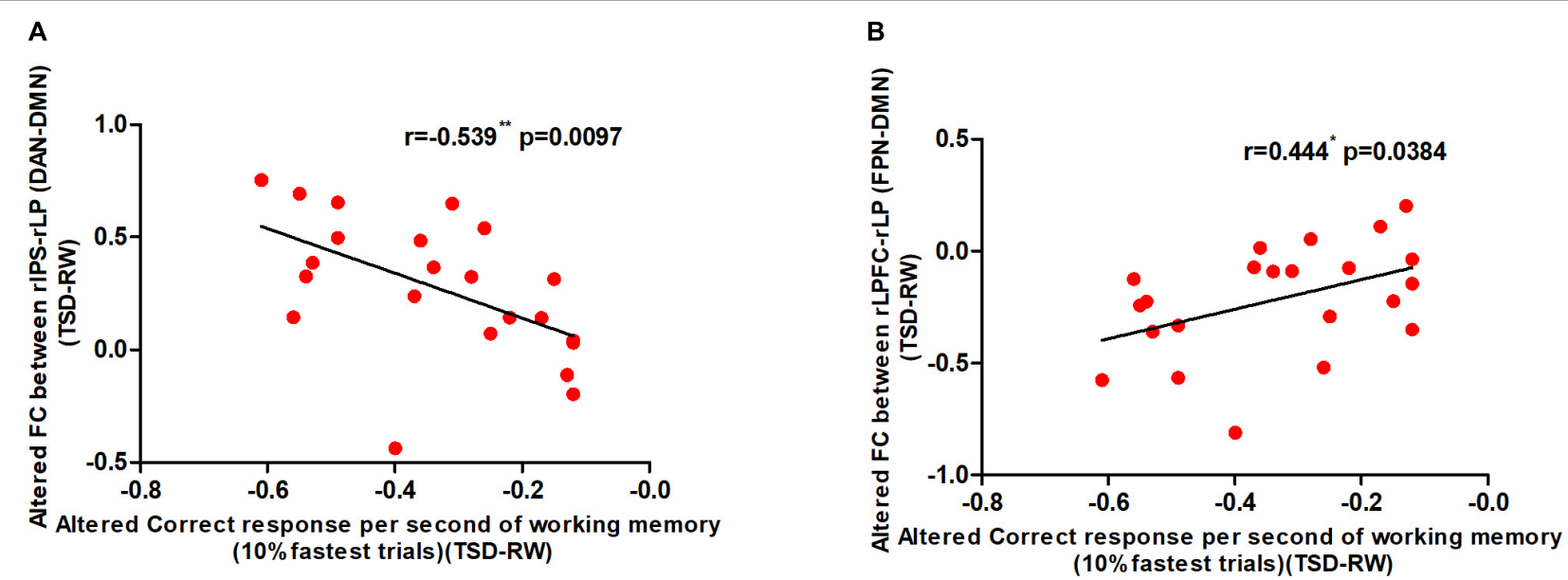

FIGURE 6 | Alerted functional connectivity correlate to altered correct response time of working memory: Alerted correct response per second of working memory is (A) negatively correlated with the alerted functional connectivity of rIPS-rLP (DAN-DMN), and (B) positively correlated with the alerted functional connectivity of rLPFC-rLP (FPN-DMN) $\left[{ }^{\star} p<0.05\right.$ and $\left.{ }^{* *} p<0.01\right]$. TSD, total sleep deprivation; RW, rested wakefulness; rIPS, right intraparietal sulcus; DAN, dorsal attention network; rLP, right paracentral lobule; DMN, default mode network; rLPFC, right lateral prefrontal cortex; FPN, fronto-parietal network (10\% fastest trials of working memory). 
components, including $\mathrm{N}_{2}, \mathrm{P}_{3}$, and $\mathrm{P}_{6}$ (Mograss et al., 2008, 2009; Qi et al., 2010).

Neuroimaging studies of SD have found that many changes in cognitive function are related to changes in DMN and other brain network connections (Havas et al., 2012; Esposito et al., 2018; Jemery and Volker, 2019). Our research found similar results. WM models address situations where cognitive resources are sufficient and cognitive function is adjusted. However, it has been found that these models are affected by significant changes in attention resources after SD (Anderson and Horne, 2006; Lim and Dinges, 2010). Therefore, attentional resource factors should be added to this model to make it applicable to interpretation of generation of WM and changes in its mechanisms under a wider range of conditions. Future research should evaluate the correlation between collaborative changes in brain networks and changes in WM under different attention resources.

\section{LIMITATIONS}

The main shortcoming of this study is that it only recruited young male subjects, so its results cannot be extrapolated to women or individuals in other age groups. Previous studies have shown that effects of SD on WM can vary with age (Gerhardsson et al., 2019). Therefore, subsequent research should include subjects of both sexes and a broader range of ages. Second, although we asked the subjects to stay awake during the resting scan and took measures to keep them awake, we cannot exclude the possibility that some may have fallen asleep during the scanning procedure. If this occurred, the effect of SD will be affected. Therefore, future researchers should include EEG to obtain objective data to identify whether the subjects actually fall asleep. Third, we used only the 2-back paradigm to measure WM, which cannot analyze changes in WM load and functional connectivity within the brain network.

\section{CONCLUSION}

In short, current study observed significant changes in connections between brain networks and found a link between changes in brain networks and changes in WM after SD. This

\section{REFERENCES}

Anderson, C., and Horne, J. A. (2006). Sleepiness enhances distraction during a monotonous task. Sleep 29, 573-576. doi: 10.1093/sleep/29.4.573

Baddeley, A. (2007). Oxford Psychology Series: Vol. 45. Working Memory, Thought, and Action. Oxford: Oxford University Press, doi: 10.1093/acprof: oso/9780198528012.001.0001

Baddeley, A. (2010). Working memory. Curr. Biol. 20, R136-R140. doi: 10.1016/j. cub.2009.12.014

Baddeley, A. (2012). Working memory: theories, models, and controversies. Annu. Rev. Psychol. 63, 1-29. doi: 10.1146/annurev-psych-120710100422

Badre, D., and Wagner, A. D. (2007). Left ventrolateral prefrontal cortex and the cognitive control of memory. Neuropsychologia 45, 2883-2901. doi: 10.1016/j. neuropsychologia.2007.06.015 may help us to clarify the brain network mechanism that sleep affects WM, the relationship between different brain regions and brain networks and WM, and to treat some memory disorders.

\section{DATA AVAILABILITY STATEMENT}

The datasets generated for this study are available on request to the corresponding author.

\section{ETHICS STATEMENT}

The studies involving human participants were reviewed and approved by the Ethics Committee of The General Hospital of PLA (Beijing, China). The patients/participants provided their written informed consent to participate in this study.

\section{AUTHOR CONTRIBUTIONS}

$\mathrm{CD}$ and $\mathrm{YZ}$ conceptualized, investigated, visualized the data, carried out the formal analysis, and wrote the manuscript. $\mathrm{XC}, \mathrm{ZP}$, and $\mathrm{LZ}$ conceptualized and investigated the data. YS conceptualized and supervised the data and carried out the funding acquisition and project administration. CW conceptualized the data and carried out the funding acquisition and project administration. All authors contributed to the article and approved the submitted version.

\section{FUNDING}

This research was supported by the National Science Foundation of Winter Olympics Technology Plan of China (grant no. 2019YFF0301600) and the National Science and Technology Commission Project (grant no. 2019JCJQZD119).

\section{ACKNOWLEDGMENTS}

The authors are grateful to Haisheng Chang for his help to this research.

Basner, M., and Dinges, D. F. (2011). Maximizing sensitivity of the psychomotor vigilance test (PVT) to sleep loss. Sleep 34, 581-591. doi: 10.1093/sleep/34.5.581

Behzadi, Y., Restom, K., Liau, J., and Liu, T. T. (2007). A component based noise correction method (compcor) for bold and perfusion based fmri. Neuroimage 37, 90-101. doi: 10.1016/j.neuroimage.2007.04.042

Biswal, B. B. (2012). Resting state fMRI: a personal history. Neuroimage 62, 938-944. doi: 10.1016/j.neuroimage.2012.01.090

Blumenfeld, R., and Ranganath, C. (2007). Prefrontal cortex and long-term memory encoding: an integrative review of findings from neuropsychology and neuroimaging. Neurosci. Rev. J. Bring. Neurobiol. Neurol. Psychiatr. 13:280. doi: $10.1177 / 1073858407299290$

Boardman, J. M., Bei, B., Mellor, A., Anderson, C., Sletten, T. L., Drummond, S. P. A., et al. (2018). The ability to self-monitor cognitive performance during $60 \mathrm{~h}$ total sleep deprivation and following 2 nights recovery sleep. J. Sleep Res. 27:e12633. doi: 10.1111/jsr.12633 
Bunge, S. A., Carter, W., David, B., and Wagner, A. D. (2005). Analogical reasoning and prefrontal cortex: evidence for separable retrieval and integration mechanisms. Cereb. Cortex 15, 239-249. doi: 10.1093/cercor/bhh126

Cappell, K. A., Gmeindl, L., and Reuter-Lorenz, P. A. (2010). Age differences in prefrontal recruitment during verbal working memory maintenance depend on memory load. Cortex 46:473. doi: 10.1016/j.cortex.2009.11.009

Champod, A. S., and Petrides, M. (2010). Dissociation within the frontoparietal network in verbal working memory: a parametric functional magnetic resonance imaging study. J. Neurosci. 30, 3849-3856. doi: 10.1523/JNEUROSCI. 0097-10.2010

Chee, M. W. L., Chuah, L. Y. M., Venkatraman, V., Chan, W. Y., Philip, P., and Dinges, D. F. (2006). Functional imaging of working memory following normal sleep and after 24 and $35 \mathrm{~h}$ of sleep deprivation: correlations of frontoparietal activation with performance. Neuroimage 31, 419-428. doi: 10.1016/j. neuroimage.2005.12.001

Chein, J. M., Ravizza, S. M., and Fiez, J. A. (2003). Using neuroimaging to evaluate models of working memory and their implications for language processing. J. Neurolinguist. 16, 315-339. doi: 10.1016/S0911-6044(03)00021-6

Chen, W. H., Chen, J., Lin, X., Li, P., Shi, L., and Shi, J. (2018). Dissociable effects of sleep deprivation on functional connectivity in the dorsal and ventral default mode networks. Sleep Med. 50, 137-144. doi: 10.1016/j.sleep.2018. 05.040

Collette, F., and Linden, M. V. D. (2002). Brain imaging of the central executive component of working memory. Neurosci. Biobehav. Rev. 26, 105-125. doi: 10.1016/S0149-7634(01)00063-X

Costers, L., Van Schependom, J., Laton, J., Baijot, J., Sjøgård, M., Wens, V., et al. (2020). Spatiotemporal and spectral dynamics of multi-item working memory as revealed by the n-back task using MEG. Hum. Brain Mapp. 41, 2431-2446. doi: $10.1002 / \mathrm{hbm} .24955$

Cowan, N. (1999). “An embedded-processes model of working memory," in Models of Working Memory: Mechanisms of Active Maintenance and Executive Control, eds A. Miyake, and P. Shah (Cambridge: Cambridge University Press), 62-101. doi: 10.1017/СBO9781139174909.006

Curtis, C. E., and D'esposito, M. (2003). Persistent activity in the prefrontal cortex during working memory. Trends Cogn. Sci. 7, 415-423. doi: 10.1016/S13646613(03)00197-9

Drummond, S. P. A., Anderson, D. E., Straus, L. D., Vogel, E. K., and Perez, V. B. (2012). The effects of two types of sleep deprivation on visual working memory capacity and filtering efficiency. PLoS One 7:e35653. doi: 10.1371/journal.pone. 0035653

Dunsmoor, J. E., Murty, V. P., Davachi, L., and Phelps, E. A. (2015). Emotional learning selectively and retroactively strengthens memories for related events. Nature 520, 345-348. doi: 10.1038/nature14106

Esposito, R., Cieri, F., Chiacchiaretta, P., Cera, N., Lauriola, M., Di Giannantonio, M., et al. (2018). Modifications in resting state functional anticorrelation between default mode network and dorsal attention network: comparison among young adults, healthy elders and mild cognitive impairment patients. Brain Imag. Behav. 12, 127-141. doi: 10.1007/s11682-0179686-y

Fairhall, S. L., Indovina, I., Driver, J., and Macaluso, E. (2009). The brain network underlying serial visual search: comparing overt and covert spatial orienting, for activations and for effective connectivity. Cereb. Cortex 19, 2946-2958. doi: 10.1093/cercor/bhp064

Fornito, A., Harrison, B. J., Zalesky, A., and Simons, J. S. (2012). Competitive and cooperative dynamics of large-scale brain functional networks supporting recollection. Proc. Natl. Acad. Sci. U.S.A. 109, 12788-12793. doi: 10.1073/pnas. 1204185109

Frenda, S. J., Patihis, L., Loftus, E. F., Lewis, H. C., and Fenn, K. M. (2014). Sleep deprivation and false memories. Psychology 25, 1674-1681. doi: 10.1177/ 0956797614534694

Gerhardsson, A., Fischer, H., Lekander, M., Kecklund, G., Axelsson, J., Åkerstedt, T., et al. (2019). Positivity effect and working memory performance remains intact in older adults after sleep deprivation. Front. Psychol. 10:605. doi: 10. 3389/fpsyg.2019.00605

Greicius, M. D., Krasnow, B., Reiss, A. L., and Menon, V. (2003). Functional connectivity in the resting brain: a network analysis of the default mode hypothesis. Proc. Natl. Acad. Sci. U.S.A. 100, 253-258. doi: 10.1073/pnas. 0135058100
Gujar, N., Yoo, S. S., Hu, P., and Walker, M. P. (2010). The unrested resting brain: sleep deprivation alters activity within the default-mode network. J. Cogn. Neurosci. 22, 1637-1648. doi: 10.1162/jocn.2009.21331

Harrington, M. O., Nedberge, K. M., and Durrant, S. J. (2018). The effect of sleep deprivation on emotional memory consolidation in participants reporting depressive symptoms. Neurobiol. Learn. Mem. 152:13.

Havas, J. A. D., Parimal, S., Soon, C. S., and Chee, M. W. L. (2012). Sleep deprivation reduces default mode network connectivity and anti-correlation during rest and task performance. Neuroimage 59, 1745-1751. doi: 10.1016/j.neuroimage.2011. 08.026

Heuvel, M. P. V. D., and Pol, H. E. H. (2010). Exploring the brain network: a review on resting-state fMRI functional connectivity. Eur. Neuropsychopharmacol. 20, 519-534. doi: 10.1016/j.euroneuro.2010.03.008

Jansma, J. M., Ramsey, N. F., Coppola, R., and René, S. K. (2000). Specific versus nonspecific brain activity in a parametric n-back task. Neuroimage 12, 688-697. doi: 10.1006/nimg.2000.0645

Jemery, M. T., and Volker, N. (2019). Cortico-limbic pain mechanisms. Neurosci. Lett. 702, 15-23. doi: 10.1016/jneulet.2018.11.037

Jones, K., and Harrison, Y. (2001). Frontal lobe function, sleep loss and fragmented sleep. Sleep Med. Rev. 5:475. doi: 10.1053/smrv.2001.0203

Kusztor, A., Raud, L., Juel, B. E., Nilsen, A. S., Storm, J. F., and Huster, R. J. (2019). Sleep deprivation differentially affects subcomponents of cognitive control. Sleep 42:zsz016. doi: 10.1093/sleep/zsz016

Lei, X., Zhao, Z., and Chen, H. (2013). Extraversion is encoded by scale-free dynamics of default mode network. Neuroimage 74, 52-57. doi: 10.1016/j. neuroimage.2013.02.020

Li, M., Gao, Y., Gao, F., Anderson, A. W., Ding, Z., and Gore, J. C. (2020). Functional engagement of white matter in resting-state brain networks. Neuroimage 220:117096. doi: 10.1016/j.neuroimage.2020.117096

Li, N., Zhang, J. M., and Liu, X. Y. (2014). Research of EEG features induced by the effects of sleep deprivation on vision short-term memory. Modern Instrum. Med. Treat. 20, 5-8. doi: 10.11876/mimt201401002

Lim, J., and Dinges, D. F. (2010). A meta-analysis of the impact of short-term sleep deprivation on cognitive variables. Psychol. Bull. 136, 375-389. doi: 10.1037/ a0018883

Lu, F. M., Cui, Q., Huang, X. J., Li, L. Y., Duan, X. J., and Chen, H. F. (2020). Anomalous intrinsic connectivity within and between visual and auditory networks in major depressive disorder. Prog. Neuro Psychopharmacol. Biol. Psychiatry 100:109889. doi: 10.1016/j.pnpbp.2020.109889

$\mathrm{Lu}, \mathrm{L}$. (2016). The effect of $72 \mathrm{~h}$ of sleep deprivation on the iowa gambling task. Noropsikiyatri Arsivi 53:357. doi: 10.5152/npa.2016.12505

Luerding, R., Weigand, T., Bogdahn, U., and Schmidt-Wilcke, T. (2008). Working memory performance is correlated with local brain morphology in the medial frontal and anterior cingulate cortex in fibromyalgia patients: structural correlates of pain-cognition interaction. Brain 131(Pt 12), 3222-3231. doi: 10. 1093/brain/awn229

Martínez-Cancino, D. P., Azpiroz-Leehan, J., and Jiménez-Angeles, L. (2015). The effects of sleep deprivation in working memory using the n-back task. IFMBE Proc. 49, 421-424. doi: 10.1007/978-3-319-13117-7_108

Mograss, M. A., Guillem, F., Brazzini-Poisson, V., and Godbout, R. (2009). The effects of total sleep deprivation on recognition memory processes: a study of event-related potential. Neurobiol. Learn. Mem. 91, 343-352. doi: 10.1016/j.nlm. 2009.01.008

Mograss, M. A., Guillem, F., and Godbout, R. (2008). Event-related potentials differentiates the processes involved in the effects of sleep on recognition memory. Psychophysiology 45, 420-434. doi: 10.1111/j.1469-8986.2007.00643.x

Moran, J. M., Kelley, W. M., and Heatherton, T. F. (2013). What can the organization of the brain's default mode network tell us about self-knowledge? Front. Hum. Neurosci. 7:391. doi: 10.3389/fnhum.2013.00391

Mu, Q., Nahas, Z., Johnson, K. A., Yamanaka, K., Mishory, A., Koola, J., et al. (2005). Decreased cortical response to verbal working memory following sleep deprivation. Sleep 28, 55-67. doi: 10.1093/sleep/28.1.55

Oberauer, K. (2002). Access to information in working memory: exploring the focus of attention. J. Exper. Psychol. Learn. Mem. Cogn. 28, 411-421. doi: 10. 1037/0278-7393.28.3.411

Owen, A. M., McMillan, K. M., Laird, A. R., and Bullmore, E. (2005). Nback working memory paradigm: a meta-analysis of normative functional neuroimaging studies. Hum. Brain Mapp. 25, 46-59. doi: 10.1002/hbm.20131 
Preece, D. (2012). The effect of working memory (n-back) training on fluid intelligence. Front. Hum. Neurosci. 6, 81-89. doi: 10.3389/fnhum.2012.00166

Ptak, R., and Schnider, A. (2010). The dorsal attention network mediates orienting toward behaviorally relevant stimuli in spatial neglect. J. Neurosci. 30, 1255712565. doi: 10.1523/JNEUROSCI.2722-10.2010

Qi, J. L., Shao, Y. C., Miao, D., Fan, M., Bi, G. H., and Yang, Z. (2010). The effects of 43 hours of sleep deprivation on executive control functions: event-related potentials in a visual go/no go task. Soc. Behav. Person. Intern. J. 38, 29-42. doi: 10.2224/sbp.2010.38.1.29

Qin, P., and Northoff, G. (2011). How is our self related to midline regions and the default-mode network? Neuroimage 57, 1221-1233. doi: 10.1016/j.neuroimage. 2011.05.028

Roberts, B. M., Libby, L. A., Inhoff, M. C., and Ranganath, C. (2017). Brain activity related to working memory for temporal order and object information. Behav. Brain Res. 354, 55-63. doi: 10.1016/j.bbr.2017.05.068

Sakagami, M., and Tsutsui, K. (1999). The hierarchical organization of decision making in the primate prefrontal cortex. Neurosci. Res. 34:79. doi: 10.1016/ S0168-0102(99)00038-3

Schilbach, L., Eickhoff, S. B., Rotarska-Jagiela, A., Fink, G. R., and Vogeley, K. (2008). Minds at rest? social cognition as the default mode of cognizing and its putative relationship to the "default system" of the brain. Conscious. Cogn. 17, 457-467. doi: 10.1016/j.concog.2008.03.013

Seidman, L. J., Thermenos, H. W., Poldrack, R. A., Peace, N. K., Koch, J. K., and Tsuang, M. T. (2006). Altered brain activation in dorsolateral prefrontal cortex in adolescents and young adults at genetic risk for schizophrenia: an fmri study of working memory. Schizophr. Res. 85, 58-72. doi: 10.1016/j.schres.2006. 03.019

Seo, J., Kim, S. H., Kim, Y. T., Song, H. J., Lee, J. J., Kim, S. H., et al. (2012). Working memory impairment in fibromyalgia patients associated with altered frontoparietal memory network. PLoS One 7:e37808. doi: 10.1371/journal.pone. 0037808

Soveri, A., Karlsson, E. P. A., Waris, O., Grönholm-Nyman, P., and Laine, M. (2017). Pattern of near transfer effects following working memory training with a dual n-back task. Exper. Psychol. 64, 240-252. doi: 10.1027/1618-3169/ a000370

Tamber-Rosenau, B. J., Asplund, C. L., and Rene, M. (2018). Functional dissociation of the inferior frontal junction from the dorsal attention network in top-down attentional control. J. Neurophysiol. 120, 2498-2512. doi: 10.1152/ jn. 00506.2018

Tempesta, D., Socci, V., Dello Ioio, G., De Gennaro, L., and Ferrara, M. (2017). The effect of sleep deprivation on retrieval of emotional memory: a behavioural study using film stimuli. Exper. Brain Res. 235, 3059-3067. doi: 10.1007/s00221017-5043-z
Terán-Pérez, G. J., Ruiz-Contreras, A. E., González-Robles, R. O., TarragoCastellanos, R., Mercadillo, R. E., Jiménez-Anguiano, A., et al. (2012). Sleep deprivation affects working memory in low but not in high complexity for the N-Back test. Neurosci. Med. 03, 380-386. doi: 10.4236/nm.2012. 34047

Van, D. E. V., Jan-Willem, T., Atsuko, T., Markus, B., Fernández Guillén, L., and Kim, F. (2012). Sleep supports selective retention of associative memories based on relevance for future utilization. PLoS One 7:e43426. doi: 10.1371/journal. pone. 0043426

Wang, L., Liu, X., Guise, K. G., Knight, R. T., Ghajar, J., and Fan, J. (2010). Effective connectivity of the fronto-parietal network during attentional control. J. Cogn. Neurosci. 22, 543-553. doi: 10.1162/jocn.2009. 21210

Whitfield-Gabrieli, S., and Nieto-Castanon, A. (2012). Conn: a functional connectivity toolbox for correlated and anticorrelated brain networks. Brain Connect. 2, 125-141. doi: 10.1089/brain.2012.0073

Wu, G., Wang, Y., Mwansisya, T. E., Pu, W., and Zhang, H. (2014). Effective connectivity of the posterior cingulate and medial prefrontal cortices relates to working memory impairment in schizophrenic and bipolar patients. Schizophr. Res. 158, 85-90. doi: 10.1016/j.schres.2014.06.033

Yeung, M. K., Lee, T. L., Cheung, W. K., and Chan, A. S. (2018). Frontal underactivation during working memory processing in adults with acute partial sleep deprivation: a near-infrared spectroscopy study. Front. Psychol. 9:742. doi: 10.3389/fpsyg.2018.00742

Yoon, J. H., Grandelis, A., and Maddock, R. J. (2016). Dorsolateral prefrontal cortex gaba concentration in humans predicts working memory load processing capacity. J. Neurosci. 36, 11788-11794. doi: 10.1523/JNEUROSCI.1970-16. 2016

Zhu, W., Chen, Q., Xia, L., Beaty, R. E., Yang, W., Tian, F., et al. (2017). Common and distinct brain networks underlying verbal and visual creativity. Hum. Brain Mapp. 38, 2094-2111. doi: 10.1002/hbm.23507

Conflict of Interest: The authors declare that the research was conducted in the absence of any commercial or financial relationships that could be construed as a potential conflict of interest.

Copyright (c) 2020 Dai, Zhang, Cai, Peng, Zhang, Shao and Wang. This is an openaccess article distributed under the terms of the Creative Commons Attribution License (CC BY). The use, distribution or reproduction in other forums is permitted, provided the original author(s) and the copyright owner(s) are credited and that the original publication in this journal is cited, in accordance with accepted academic practice. No use, distribution or reproduction is permitted which does not comply with these terms. 